\title{
Patrice BRODEUR
}

Professeur à la faculté de théologie et de sciences des religions de l'Université de Montréal, titulaire de la chaire islam, pluralisme et globalisation

\section{(2008)}

\section{"La commission Bouchard-Taylor et la perception des rapports entre «uébécois» et « musulmans » au Québec."}

Un document produit en version numérique par Jean-Marie Tremblay, bénévole, professeur de sociologie retraité du Cégep de Chicoutimi

Courriel: jean-marie tremblay@uqac.ca

Site web pédagogique : $h$ ttp://www.uqac.ca/jmt-sociologue/

Dans le cadre de: "Les classiques des sciences sociales" Une bibliothèque numérique fondée et dirigée par Jean-Marie Tremblay, professeur de sociologie au Cégep de Chicoutimi

Site web: http://classiques.uqac.ca/

Une collection développée en collaboration avec la Bibliothèque

Paul-Émile-Boulet de l'Université du Québec à Chicoutimi

Site web: $h+t p: / /$ bibliotheque.uqac.ca/ 


\section{Politique d'utilisation de la bibliothèque des Classiques}

Toute reproduction et rediffusion de nos fichiers est interdite, même avec la mention de leur provenance, sans l'autorisation formelle, écrite, du fondateur des Classiques des sciences sociales, Jean-Marie Tremblay, sociologue.

Les fichiers des Classiques des sciences sociales ne peuvent sans autorisation formelle:

- être hébergés (en fichier ou page web, en totalité ou en partie) sur un serveur autre que celui des Classiques.

- servir de base de travail à un autre fichier modifié ensuite par tout autre moyen (couleur, police, mise en page, extraits, support, etc...),

Les fichiers (.html, .doc, .pdf, .rtf, .jpg, .gif) disponibles sur le site Les Classiques des sciences sociales sont la propriété des Classiques des sciences sociales, un organisme à but non lucratif composé exclusivement de bénévoles.

Ils sont disponibles pour une utilisation intellectuelle et personnelle et, en aucun cas, commerciale. Toute utilisation à des fins commerciales des fichiers sur ce site est strictement interdite et toute rediffusion est également strictement interdite.

L'accès à notre travail est libre et gratuit à tous les utilisateurs. C'est notre mission.

Jean-Marie Tremblay, sociologue

Fondateur et Président-directeur général, LES CLASSIQUES DES SCIENCES SOCIALES. 
Cette édition électronique a été réalisée par Jean-Marie Tremblay, bénévole, professeur de sociologie au Cégep de Chicoutimi à partir de :

\section{Patrice BRODEUR}

"La commission Bouchard-Taylor et la perception des rapports entre « Québécois « et « musulmans » au Québec".

Un article publié dans la revue Cahiers de recherche sociologique, no 46, septembre 2008, pp. 95-107. Montréal : Département de sociologie, UQAM. Liber, Éditeur. Un numéro intitulé : "L'islam, I'Empire et la République."

[Autorisation formelle accordée le 7 septembre 2010 par Monsieur Rachad Antonius, directeur du numéro, de diffuser tous les articles de ce numéro de la revue dans Les Classiques des sciences sociales.]

Courriels: antonius.rachad@uqam.ca

patrice.brodeur@umontreal.ca

Polices de caractères utilisée : Comic Sans, 12 points.

Édition électronique réalisée avec le traitement de textes Microsoft Word 2008 pour Macintosh.

Mise en page sur papier format : LETTRE US, $8.5^{\prime \prime} \times 11^{\prime \prime}$.

Édition numérique réalisée le 12 décembre 2011 à Chicoutimi, Ville de Saguenay, Québec. 


\section{Patrice BRODEUR}

"La commission Bouchard-Taylor et la perception des rapports entre «Québécois» et « musulmans » au Québec".
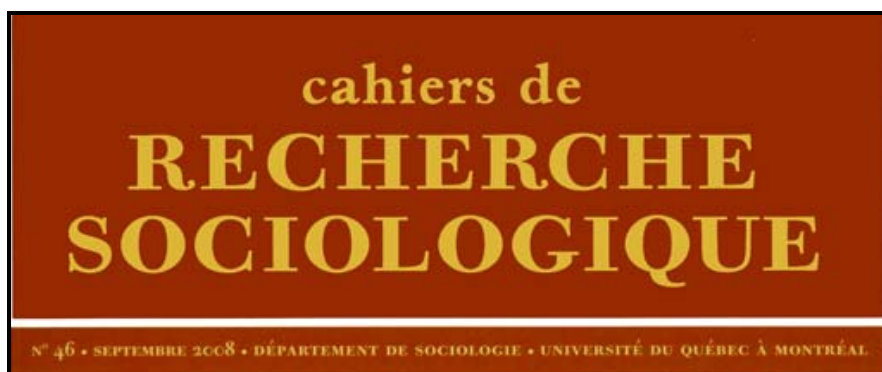

\section{L'islam, l'Empire et la République}

\section{Liber}

Un article publié dans la revue Cahiers de recherche sociologique, no 46, septembre 2008, pp. 95-107. Montréal : Département de sociologie, UQAM. Liber, Éditeur. Un numéro intitulé : "L'islam, l'Empire et la République." 
Patrice Brodeur. Professeur à la faculté de théologie et de sciences des religions de l'université de Montréal, titulaire de la chaire islam, pluralisme et globalisation.

patrice.brodeur@umontreal.ca

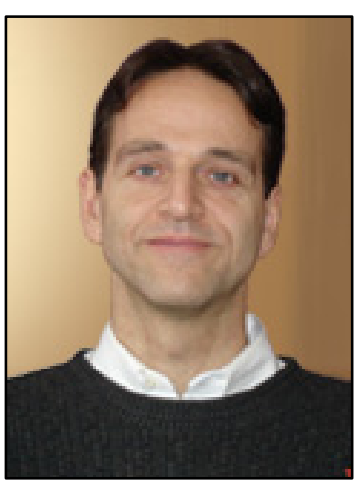




\section{Table des matières}

Introduction

L'approche pluriscope

Remarques contextuelles

Contexte géopolitique et diversité identitaire

Triple héritage historique et contemporain

Des questions à poser

Pistes de réflexion

Hypothèse de recherche

Résumé / Abstract / Resumen 


\section{Patrice BRODEUR}

"La commission Bouchard-Taylor et la perception des rapports entre « Québécois » et « musulmans » au Québec".

Un article publié dans la revue Cahiers de recherche sociologique, no 46, septembre 2008, pp. 95-107. Montréal : Département de sociologie, UQAM. Liber, Éditeur. Un numéro intitulé : "L'islam, l'Empire et la République."

\section{Introduction}

\section{Retour à la table des matières}

Cet article jette un premier regard théorique sur la façon de comprendre la commission Bouchard-Taylor, dont le rapport final a été publié le 22 mai 2008, et en particulier sur la place que l'islam a occupée dans l'espace imaginaire qu'elle a créé au Québec. Cet espace est constitué autant de discours écrits qu'oraux, dont seul une partie minime est accessible aux chercheurs. En effet, le gros des conversations du grand public qu'a engendrées cette commission n'a jamais été transcrit ou enregistré. Il nous reste quand même les heures de films tirées des trente et un jours d'audiences publiques à travers le Québec et des quatre forums nationaux, des interviews télévisés et radiophoniques multiples, les textes de la commission elle-même et surtout son rapport final ${ }^{1}$, les articles des journaux et revues ainsi que ceux

$1 G$. Bouchard et $C$. Taylor, Fonder l'avenir : le temps de la conciliation, rapport de la Commission de consultation sur les pratiques d'accommodement reliées aux différences culturelles, gouvernement du Québec, mai 2008, http://www.accommodements.qc.ca/commission/index.html. 
encore accessibles sur le site internet de la commission et, enfin, toutes les notes privées des membres de cette commission - à supposer qu'elles seront un jour mises à la disposition du public. Bref, ce riche corpus devrait nous aider à mieux comprendre les réactions de personnes et de groupes sur les questions d'accommodements raisonnables et d'ajustements concertés.

Dans cet article, je propose d'aborder en cinq sections la perception des rapports entre «Québécois» et «musulmans» à la lumière des événements reliés à la commission Bouchard-Taylor: une balise théorique, des remarques contextuelles, des questions pour une analyse future, ainsi que [96] des pistes de réflexion et une hypothèse de recherche. Il ne s'agit donc pas ici d'une analyse de tout ce riche corpus, travail plus considérable qui exigera beaucoup plus de temps, mais bien d'un premier regard théorique rédigé pendant que la poussière retombe lentement sur ce sujet fascinant qui a retenu l'attention du Québec la dernière année et demie.

\section{L'approche pluriscope}

Retour à la table des matières

La balise théorique proposée pour cette première réflexion provient d'une approche pluriscope double : les identités pluriscopes et les géographies pluriscopes. "Une identité pluriscope est une identité dont l'interprétation change selon les différents points de vue possibles provenant des autres identités qui s'y rattachent. Selon la nature plus ou moins changeante des moments politiques, nécessitant de constants réaménagements des intérêts de chaque groupe qui façonne les dynamiques de pouvoir de toutes sortes, les identités varient, changent de l'intérieur comme de l'extérieur, constamment influencées par le rapport qu'elles entretiennent entre elles. En fait, ces identités et surtout leurs significations sont fluides parce qu'elles sont ellesmêmes constitutives les unes des autres, reflétant une grande interdépendance. [...] En parallèle au concept d'identités pluriscopes, je propose le concept de géographies pluriscopes puisque bien des identités au coeur des débats politiques contemporains, mais pas toutes, se 
réfèrent implicitement ou explicitement à des référents géographiques. Les représentations linguistiques et interprétations herméneutiques de la géographie des espaces planétaires sont tout aussi pluriscopes que celles des identités. [...] Les processus de géographies pluriscopes sont plus ou moins visibles selon différents contextes politiques ${ }^{2}$. »

Cette approche théorique des identités et géographies pluriscopes permet de mieux analyser l'interdépendance des référents identitaires et géographiques explicites ou implicites qui sous-tendaient les arguments présentés à la commission Bouchard-Taylor ainsi que leur interpénétration : c'est le « Nous » juxtaposé dont il est question dans le rapport final. Une autre représentation qu'on peut s'en donner est celle des poupées russes. Si cette analogie est bien adaptée aux géographies pluriscopes - car les éléments géographiques sont souvent emboîtés les uns dans les autres, du plus petit référent géographique (un « chez moi » où l'on habite le plus souvent) au plus grand (l'univers ) - elle l'est moins aux identités pluriscopes. La limite principale de l'analogie est que, même si elles sont parfois emboîtables particulièrement lorsqu'elles sont directement reliées à des lieux, les identités pluriscopes dépassent en général tout référent géographique. Ce qui veut dire qu'elles sont d'abord fluides et interchangeables, [97] surtout celles qui remontent à la conscience à des moments précis. Ces moments valent également la peine d'être bien définis, comme d'ailleurs la personne qui en fait l'analyse ${ }^{3}$.

Les deux mots associés à notre sujet, «Québécois» et «musulman », sont l'un et l'autre directement liés à des identités pluriscopes,

2 P. Brodeur, «Les tensions religieuses et internationales au Moyen-Orient : existe-t-il une menace islamiste? », dans R. Crépeau (dir.), Religions et politiques internationales, Montréal, Presses de l'université de Montréal, à paraître en 2009.

3 L'auteur de cet article se définit comme Québécois non musulman, faisant partie du groupe identitaire majoritaire au Québec et minoritaire au Canada des Québécois canadiens-français. Il est également chercheur, principalement dans le domaine de l'islam contemporain. Étant retourné au Québec, après quinze ans d'absence, au moment même du débat sur la question des cours d'arbitrage islamique en Ontario en septembre 2005, il est très conscient d'être revenu dans une société québécoise et canadienne transformée par un accroissement de l'immigration de personnes de religion et de cultures islamiques. 
elles-mêmes liées aux géographies pluriscopes, quoique, dans le cas de "Québécois», de manière plus évidente que dans celui de «musulman». Dans ce dernier cas, il faut expliciter le fait que le mot « musulman » est très souvent implicitement rattaché, tant par les musulmans que par les non-musulmans, à une géographie pluriscope du «monde musulman», en référence aux lieux où sont regroupés les pays dont la majorité des habitants sont musulmans.

Enfin, la distinction entre ces deux termes n'implique pas qu'ils soient mutuellement exclusifs. Plusieurs musulmans au Québec, la grande majorité peut-être, se disent «musulmans québécois» ou «Québécois musulmans», selon l'importance qu'ils veulent donner à l'une ou l'autre de ces deux identités, que chaque personne vise à intégrer à sa façon. Mais pour ne pas alourdir le texte, le mot «Québécois» sera utilisé dans cet article comme signifiant Québécois non musulman du groupe ethnique majoritaire canadien-français, et « musulman », comme signifiant musulman vivant au Québec.

\section{Remarques contextuelles}

\section{Retour à la table des matières}

Nos remarques contextuelles sont de deux ordres. D'abord, il y a le contexte géopolitique du Québec, qui est partagé par toutes les personnes incluses dans les discussions de la commission Bouchard-Taylor, tant «Québécois » non musulmans que «musulmans » vivant au Québec, qu'elles se disent «musulmans québécois», "Québécois musulmans », ou encore « musulmans » ou « Québécois »... Puis, un triple héritage caractérise le contexte pluriscope à l'intérieur duquel on doit replacer les interactions de la commission Bouchard-Taylor: I'histoire des rapports entre Église et société au Québec depuis la Révolution tranquille et le processus de sécularisation des institutions publiques et d'une partie de la population: l'histoire des relations islamochrétiennes depuis l'époque médiévale et surtout islamo-occidentales depuis le 11 septembre 2001 ; et la situation politique dans laquelle s'est réalisée la mise sur pied de la commission Bouchard-Taylor. 
[98]

\section{Contexte géopolitique et diversité identitaire}

\section{Retour à la table des matières}

Tout comme il est normal de penser l'identité québécoise - quelle qu'en soit la définition - comme une intégration plus ou moins réussie d'une diversité de groupes identitaires (régions, généalogies, dialectes, croyances, idéologies, etc.), il en va de même, à plus forte raison, en ce qui concerne la diversité au sein de l'identité musulmane (plus d'un milliard d'individus de par le monde laissent place à beaucoup plus de diversité que sept millions) ${ }^{4}$.

Sociologiquement, les musulmans du Québec sont en grande majorité sunnites, ce qui est conforme aux proportions dans la population musulmane mondiale. Mais il existe aussi plusieurs groupes chiites (duodécimains et septimains en particulier), ainsi que quelques membres de la communauté Ahmadiya (ou Qadiyani), jugés hétérodoxes tant par les sunnites que par les chiites. Ces différences sociologiques s'expriment principalement dans la façon dont sont organisées ces communautés au niveau de leur structure de décision et de représentation dans la société, la plupart de ces groupes identitaires ayant des structures très décentralisées.

Sur le plan juridique, il existe quatre écoles traditionnelles chez les sunnites. Quant aux chiites, chaque groupe a développé sa propre école au cours des siècles. Au sein de ces écoles, mais différemment pour chacune d'elles, il existe une grande diversité herméneutique du côté théologico-philosophique, par exemple, l'approche du mouvement des Mu'tazila (philosophie rationaliste influencée par la philosophie hellénistique), du kalâm (théologie en partie rationaliste, également influencée par la philosophie hellénistique, mais moins fortement) et plusieurs écoles modernes (littéralistes fondamentalistes, libérales, historicocritiques, progressistes, etc.). Ces écoles se réclament souvent, mais

4 Pour d'autres aspects de cette diversité, voir M.-E. Martel (dir.), Québécois et musulmans main dans la main pour la paix, Montréal, Lanctôt, 2006. 
pas toujours, d'idéologies politiques modernes issues de l'Occident, avec leurs différentes nuances de la gauche à la droite, de l'intégrisme au libéralisme, du militantisme au pacifisme, en passant par l'indifférence. Enfin, il existe les nombreux ordres mystiques (taríqât) qui offrent très souvent, mais pas forcément, des structures parallèles à celles déjà mentionnées afin de promouvoir l'enseignement et la pratique de l'islam selon une interprétation mystique. Il y a donc aujourd'hui une très grande variété d'herméneutiques tant juridiques qu'idéologiques qui s'entrecoupent à l'intérieur des communautés musulmanes.

Un autre aspect de la diversité est celui de la fréquence des pratiques rituelles. Bien que nous manquions de statistiques crédibles sur ce point, il semble que la majorité des musulmans du Québec ne pratiquent pas, ou très peu, ce qui traduit un grand degré de sécularisation. Cela s'expliquerait par les conditions particulières d'un grand nombre d'immigrants d'origine maghrébine dont beaucoup, depuis près de vingt ans, viennent au Québec justement pour échapper au poids d'une certaine islamisation salafiste.

[99]

Enfin, il existe une diversité linguistique et culturelle importante chez les musulmans du Québec, même si plus de la moitié sont d'origine maghrébine. C'est sans doute cette diversité qui explique la multiplication de mosquées et de musallahs (salle de prières) : on en compte plus de soixante-cinq dans la grande région de Montréal. La diversité est également géographique, ce qui porte à penser qu'il n'y a pas, à Montréal, la ghettoïsation que connaissent certaines grandes agglomérations européennes.

Tout cela reflète les dimensions pluriscopes qu'il faut toujours associer au terme «musulman», car l'objet de chacune d'elles n'est jamais le même pour chaque personne en tout temps, et encore moins pour la perception de l'autre à l'extérieur du groupe identitaire de référence de cette perception. L'identité pluriscope à partir du mot «musulman» est à la fois source d'explications du questionnement qu'ont traduit les musulmans lors de la commission Bouchard-Taylor et source d'un questionnement identitaire pour un grand nombre de musulmans et de non-musulmans appelés à reconnaître cette diversité 
comme une sorte de miroir. Ce questionnement n'en est encore qu'à ses balbutiements et fait partie, lui aussi, de ces dynamiques identitaires qui cherchent à comprendre comment mieux vivre ensemble. Car les processus identitaires sont essentiellement relationnels : l'identité se forme dans le jeu des négociations interidentitaires, avec toutes les dynamiques de pouvoir que l'on connaît, notamment entre majorités et minorités, qui s'emboîtent les unes dans les autres ${ }^{5}$.

\section{Triple héritage historique et contemporain}

\section{Retour à la table des matières}

Pour parler du passé, du présent et de l'avenir des relations entre musulmans et non-musulmans au Québec, il faut élargir le cadre historique et géographique pour y inclure au moins trois périodes : le Moyen Âge des relations islamo-chrétiennes, celui des relations entre Églises et États et, enfin, le siècle des Lumières et de la construction des États-nations modernes. Comme un archéologue qui creuse à travers des strates pour trouver des traces du passé, l'analyste interdisciplinaire d'aujourd'hui ne peut que débuter par un travail d'historien des idées, dont le présent préserve différentes traces. En voici quelquesunes.

Histoire des rapports Église-société. Il semble que, des deux premières périodes mentionnées, subsistent deux sentiments forts: la peur des musulmans conquérants et violents héritée de l'époque des Croisades et des attaques ottomanes sur Vienne en particulier et la peur, héritée des débuts de l'ère moderne, que la religion puisse à nouveau contrôler la sphère publique. Ces peurs sont encore très réelles dans la population québécoise. La première a été en partie ravivée par le terrorisme islamiste, surtout depuis le 11 septembre 2001, et insérée dans une représentation de l'histoire [100] occidentale où les Croisades ont une place relativement positive. La seconde peur est encore plus grande : il faut la mettre en relation avec la modernisation accélé-

5 J. Heinrich et V. Dufour, Circus quebecus: Sous le chapiteau de la commission Bouchard-Taylor, Montréal, Boréal, 2008. 
rée qu'a connue le Québec depuis un demi-siècle, mais l'Occident dans son ensemble également. Or, si cela fait partie de l'expérience directe des deux générations les plus âgées de la population québécoise, les deux plus jeunes n'ont pas vécu directement cette époque réelle du contrôle de l'Église (particulièrement catholique romaine) sur la population. C'est ce qui explique en grande partie l'écart générationnel au Québec en ce qui concerne, entre autres, la perception de l'islam. Plusieurs sondages l'ont démontré.

À ce deuxième héritage, il faut ajouter divers compromis politiques qui, entre le dix-huitième et le vingtième siècle, ont marqué la relation entre les Églises et les pouvoirs politiques européens au cours de la construction des démocraties laïques. Il en résulte une série d'exceptions et d'incohérences, économiques, juridiques et même constitutionnelles, dans le traitement de différents groupes identitaires, normalement minoritaires d'une façon ou d'une autre - autochtones et Noirs, surtout en Amérique du Nord, mais aussi groupes religieux, tels les juifs, les Églises de la paix, les mormons, les premiers musulmans, etc. Au Canada, ces exceptions sont utilisées pour expliquer, voire justifier, le traitement différencié des autochtones, des minorités ici francophones là anglophones, ainsi que divers groupes religieux (catholiques, protestants, juifs et, plus récemment, musulmans), surtout dans le domaine de l'éducation.

Or, avec l'adoption de chartes des droits et libertés individuels, il y a eu multiplication de demandes de reconnaissance de sa différence, si bien que l'interprétation habituelle des normes sociales a dû être revue, ce qui a réveillé des peurs dans l'imaginaire collectif du groupe identitaire majoritaire, tant au Canada qu'au Québec. Dans le cas des réactions aux accommodements raisonnables, il y a eu ainsi amalgame de la peur des musulmans et de la peur de la religion dans l'espace public, ce qui est loin d'être la réalité dans les faits puisque la majorité des demandes d'accommodements raisonnables ne viennent pas des musulmans mais de "Québécois de souche » convertis à de nouvelles expressions chrétiennes ou d'origine chrétienne en terre québécoise (par exemple, le christianisme protestant évangélique, le christianisme 
pentecôtiste, les témoins de Jéhovah et les mormons) ${ }^{6}$. Ces demandes particulières doivent être comprises par opposition à divers discours d'intégration au sein d'un groupe identitaire commun en apparence : les «Québécois». Les groupes demandeurs de reconnaissance, qu'ils soient idéologiquement conservateurs ou non, forment un phénomène transnational qui a de plus en plus d'influence sur le positionnement social et sur les définitions de soi-même des acteurs à l'intérieur de chaque État-nation.

[101]

Rapports entre Occidentaux musulmans et non musulmans. Le Québec, le Canada et plusieurs autres pays occidentaux ont dernièrement connu des tensions sociales sérieuses relatives aux différences culturelles et religieuses, particulièrement celles entre musulmans et non-musulmans. Ces tensions se sont exacerbées après le 11 septembre 2001, dans la frénésie des mesures devant assurer la sécurité nationale et internationale. C'est donc dans ce contexte de relations internationales que se sont rapidement succédé, au Québec et au Canada au cours des deux dernières années, des décisions judiciaires qui ont fait couler beaucoup d'encre 7 . Elles ont affecté les relations entre mu-

6 M. McAndrew, M. Milot, J.-S. Imbeault et P. Eid (dir.), L'accommodement raisonnable et la diversité religieuse à l'école publique : normes et pratiques, Montréal, Fides, 2008.

7 Voici quelques exemples : 2 mars 2006, la Cour suprême du Canada autorise un jeune sikh québécois à porter son kirpan (couteau cérémonial) à l'école; 9 mars 2006 : La Presse publie un article dévoilant la décision de l'association des employeurs maritimes d'accommoder les camionneurs sikhs orthodoxes en leur donnant le droit de garder leur turban et de ne pas porter un casque de sécurité dans l'enceinte du port de Montréal : 2 août 2006 : la Commission des droits de la personne du Québec soutient l'École de technologie supérieure qui avait refusé de réserver un local de prière à ses étudiants musulmans ayant jugé raisonnable la politique d'offrir à ses étudiants de prier dans des salles de cours inutilisés : 7 novembre 2006 : La Presse dévoile l'accord entre le YMCA et la congrégation Yetev Lev, qui stipule que cette dernière paiera les vitres dépolies des fenêtres d'une salle d'entraînement du YMCA afin d'éviter la vue de femmes en tenue légère. Ces exemples, et quelques autres, sont tirés de l'annexe «Chronologie accommodante » du livre Circus quebecus. 
sulmans et non-musulmans de façon très négative, surtout au Québec, mais en Ontario également. L'automne 2005 a vu la publication du rapport de l'ex-procureur général de l'Ontario, Marion Boyd, qui recommandait l'adoption de cours d'arbitrage islamique inspirées de l'Acte de l'arbitrage de 1991 en Ontario, comme c'était le cas pour les catholiques et les juifs. Les protestations furent fortes et immédiates. En janvier 2007, les réactions, parfois violentes, aux caricatures danoises sur le prophète Mohammed ont poussé les tensions vers un nouveau sommet. Au Québec, ces réactions ont coïncidé avec la publication d'un article du Journal de Montréal et de la station de radio 98,5 FM selon lequel $59 \%$ des Québécois étaient racistes puis avec la parution du « code de vie» de Hérouxville, petit village de mille trois cents personnes en Mauricie, interdisant entre autres de lapider les femmes, de les brûler vives et de les exciser.

Situation politique lors de la mise sur pied de la commission Bouchard-Taylor. En réaction à ces événements, le 8 février 2007, la majorité du Parti libéral du gouvernement du Québec a décrété la mise en place d'une commission nationale sur les pratiques d'accommodements raisonnables avec un mandat que les commissaires ont décidé d'interpréter largement ${ }^{8}$. La commission Bouchard-Taylor fut créée à cause d'un manque de leadership politique du parti au pouvoir, le Parti libéral du Québec, mais peut-être encore plus par un manque d'engagement de tous les partis en faveur du respect du processus juridique en cours en matière d'accommodements raisonnables et de la [102] reconnaissance de leur importance dans l'intégration des minorités, surtout religieuses. Il aurait été facile de calmer la flambée d'islamophobie populaire, en demandant par exemple à la Commission des droits de la personne du Québec un état des demandes d'accommodements raisonnables, ce qui aurait permis de démontrer que la grande majorité provenait en fait de Québécois de souche canadienne-française. De plus, comme le même gouvernement avait déjà mis sur pied une commission dirigée par Bergman Fleury pour étudier la question des accommodements en milieu de l'éducation, il aurait suf-

8 G. Bouchard et C. Taylor, op. cit., p. 17. 
fi d'attendre son rapport et d'en tirer des conclusions juridiques. Mais, à la veille d'élection, aucun parti n'emprunta la voie honorable de la coopération multipartite contre l'alarmisme des médias. Une analyse fine de cette période précédant la commission Bouchard-Taylor nous apprendra si de tels efforts ont eu lieu et si à l'avenir une telle démarche ne permettrait pas d'éviter ou du moins d'atténuer les effets négatifs que la conjoncture de décisions juridiques et d'événements conflictuels a pu avoir sur la perception des rapports entre musulmans et non-musulmans au Québec.

Une fois mise sur pied, dotée d'un budget de cinq millions, la commission Bouchard-Taylor a créé sa propre dynamique, d'autant plus que par son existence même elle avait crée autour d'elle un vide politique. $\mathrm{Ce}$ vide facilita l'instrumentalisation, surtout politique mais aussi médiatique, du thème de l'immigration et des dangers supposés reliés surtout aux musulmans, sachant qu'environ $20 \%$ de l'immigration en 2006 étaient d'identités musulmanes, dont la moitié de l'Algérie. Le parti de l'ADQ, en particulier, joua sur ces peurs et réaffirma des valeurs conservatrices souvent reliées à l'intolérance d'identités minoritaires jugées non seulement comme " autres » mais comme incompatibles avec les valeurs dites «québécoises». Avec l'islamophobie grandissante, quelques traces de judéophobie refirent surface, ainsi que l'expression libre de commentaires homophobiques (reliés en partie au leader du Parti québécois, ouvertement homosexuel) et, de façon plus générale, xénophobiques. Et pour la première fois depuis plusieurs décennies, les élections d'avril 2007 ont porté au pouvoir un parti minoritaire, les mêmes libéraux, mais cette fois avec un nouveau parti d'opposition officielle, l'ADQ.

Cette nouvelle réalité politique, où le Parti québécois perdit plus que prévu, démontre que la population du Québec a été affectée, elle aussi, par le grand mouvement politique vers la droite qui s'est produit dans la foulée des réactions nationalistes patriotiques du monde occidental (à l'exception de l'Espagne) aux actes terroristes du 11 septembre 2001 et des autres qui ont suivi. La commission Bouchard-Taylor ne peut donc pas être analysée sans faire place à la fois à ces réalités passées et présentes dans la société québécoise et aux dynamiques transnationales qui l'affectent aujourd'hui de façon plus soutenue. 
Ce triple héritage historique et contemporain aide à comprendre le sentiment d'une perte d'identité dominante au Québec sous les pressions politiques et économiques globalisantes, réveillant chez certains de vieilles tendances à vouloir trouver un bouc émissaire. Ce concept fut mentionné [103] dans le mémoire soumis à la commission BouchardTaylor par l'équipe de la chaire de recherche du Canada Islam, pluralisme et globalisation de l'université de Montréal ${ }^{9}$. Il apparaît également dès le début du rapport final de la commission sous la rubrique «le malaise identitaire 10 ». Ce concept de bouc émissaire est une hypothèse importante à explorer pour mieux comprendre les réactions surtout négatives quant à l'islam en général, à ses formes d'extrémisme politique et d'antiféminisme en particulier.

\section{Des questions à poser}

\section{Retour à la table des matières}

À partir de cette approche pluriscope double, qui reconnaît la diversité intérieure de chaque groupe, plusieurs questions émergent: Comment mesurer l'intégration au sein d'une société plurielle? Est-ce que, à travers les interactions qui sont ressorties de la commission Bouchard-Taylor, les musulmans d'aujourd'hui forment les boucs émissaires des peurs de la majorité canadienne-française au Québec? Si c'est le cas, dans quelle mesure s'agit-il d'une reproduction de comportements et de discours collectifs du passé ? Est-ce que cette commission est réellement devenue au cours de l'automne 2007 un espace pour l'expression de ces tensions entre minorités musulmanes et majorité non musulmane au Québec? Si oui, comment cela s'est-il produit? Quel fut le rôle des divers acteurs sociaux (politiciens, journalistes, éducateurs, leaders d'opinion et de groupes de pression de toutes sor-

9 P. Brodeur (dir.), Ajustements concertés/Accommodements raisonnables: réflexion et propositions présentées à la Commission Bouchard-Taylor, chaire de recherche du Canada Islam, pluralisme et globalisation, faculté de théologie et de sciences des religions, université de Montréal, 30 novembre 2007.

10 G. Bouchard et C. Taylor, op. cit., p. 18. 
tes, etc.) ? Comment les musulmans du Québec y ont-ils réagi et comment peut-on mesurer l'impact à long terme de leurs réactions diverses? Est-ce que ceux parmi eux qui sont anti-fondamentalistes seront affectés de la même façon que les plus conservateurs? Peut-on réellement parler de cette commission comme d'un processus de grande pratique démocratique pour la construction d'un tissu social plus solide? Comment les musulmans et non-musulmans du Québec peuvent-ils participer ensemble à la transformation de leurs relations, qui se résument pour beaucoup au soi (Québécois de souche) contre l'autre (les immigrants, les musulmans, etc.), vers un « nous québécois», construisant ainsi une société réellement commune et inclusive? Est-ce que des mécanismes sociaux ont émergé au Québec pour participer aux initiatives de l'Alliance des civilisations des Nations unies, avec pour objet l'amélioration des relations islamo-occidentales? Enfin, est-il possible de faire ressortir statistiquement certaines tendances et leur impact sur la perception que les musulmans et les non-musulmans, tant en Occident que dans les pays à majorité musulmane, ont des uns et des autres?

[104]

Ces questions en soulèvent d'autres: Quel est l'ampleur des sentiments d'exclusion parmi les musulmans du Québec? Quels sont les facteurs qui les provoquent et les alimentent? Quels sont les facteurs qui les atténuent et ceux qui pourraient même les effacer? Est-ce que ces facteurs et surtout leurs réalités sociales selon les différentes périodes des derniers quatre cents ans existaient comme aujourd'hui? $\mathrm{Si}$ oui, comment les mesure-t-on historiquement et sociopsychologiquement aujourd'hui ? Comment peut-on ensuite déduire, à partir d'une telle élaboration, une grille qui nous permettrait de mieux connaître le degré des perceptions que différents groupes identitaires ont d'eux-mêmes par rapport à d'autres groupes, minoritaires ou majoritaires?

Les défis s'empilent: Comment est-ce que les musulmans et nonmusulmans du Québec peuvent-ils participer consciemment au processus de transformation de leur présent mode respectif du rapport à l'altérité dans un esprit d'interdépendance et d'ouverture curieuse à eux-mêmes et aux divers autres du Québec? Comment se développe 
ce désir d'intégration mutuelle sans lequel les dynamiques sociales, majorité et minorités, ne peuvent que sombrer dans deux excès : la ghettoïsation ou l'assimilation, tous deux stratagèmes de survie, le premier surtout collectif et le second surtout individuel. Le premier refuse de s'intégrer à l'autre dominant, souvent majoritaire; le second refuse de s'isoler, de marquer sa différence. Pour tout groupe majoritaire, comment vivre avec un peu des deux? Et surtout, comment accepter, par ajustements concertés et raisonnables, les choix de chacun conformément à la liberté qui a toujours fait la force de la société québécoise, dans son ensemble, mais pas toujours dans ses recoins? Nous sommes dans une zone grise, où il faut définir les limites de la tolérance à l'intolérance, de l'intolérance à la tolérance, du devoir de laisser à l'autre sa liberté, avec toutes ces conséquences, dans la mesure où elle n'enfreint pas notre propre liberté, surtout physiquement.

Ce questionnement tente de découvrir si le processus d'intégration des musulmans au Québec répète ou non des patterns juridiques et sociaux du passé, quant à l'exclusion de certaines minorités. Dans quelle mesure est-ce que la gestion de l'autre dans le processus de transformation de l'identité majoritaire québécoise (canadienne-française, catholique de souche, etc.) est constante ou non, par rapport à un héritage donné du processus d'inclusion ou d'exclusion de l'autre? La commission Bouchard-Taylor ne sert que de prisme, car elle a suscité des débats à la fois théoriques et pratiques, en particulier sur la place de l'islam dans la société québécoise. Si les musulmans au Québec font l'objet de pratiques discriminatoires déjà pratiquées de par le passé à l'égard d'autres groupes minoritaires récemment immigrés ou à « intégrer » (c'est-à-dire les autochtones, l'exclusion des Français par les Anglais, l'exclusion des autochtones par les Français, etc.), alors on peut s'attendre à ce que les résultats de cette commission produisent à la fois l'un ou l'autre de deux scénarios opposés : une plus grande intégration de la majorité des musulmans du Québec à la société majoritaire ou une plus grande ghettoïsation. Il se peut fort bien que nous assistions à un mélange [105] des deux, selon les trajectoires déjà sensibles au Québec. D'une part, les alarmistes veulent à tout prix éviter que n'apparaisse au Québec et au Canada le phénomène des banlieues parisiennes ou des caricatures danoises. D'autre part, les intégrateurs veulent à tout prix éviter de brandir les dangers d'une aliéna- 
tion réelle des musulmans du Québec à la suite de la commission Bouchard-Taylor de crainte d'envenimer la situation auprès de ces groupes minoritaires.

\section{Pistes de réflexion}

Retour à la table des matières

Devant ces scénarios, il semble utile de proposer quelques pistes de réflexion. Est-ce que le processus de relations à l'autre se reproduit de la même façon de génération en génération, même si l'identité de l'autre change une fois qu'il s'est bien intégré à la majorité ? Si c'est le cas, est-ce que le processus identitaire de la majorité aura toujours besoin d'un nouvel autre, à chaque vague successive d'immigration? Quand peut-on espérer qu'un tel cycle s'estompe pour faire place à une réelle gestion pluraliste de nos identités multiples, au Québec comme dans n'importe quelle société plurielle? Pour ce faire, ne faudra-t-il pas d'abord combattre l'insécurité face à l'autre qui est au coeur même du processus identitaire, dans ce cas-ci québécois à l'échelle du Québec et musulman à l'échelle mondiale, toujours aux prises avec le danger de manipulation émotive et politique relié à l'utilisation de boucs émissaires? En effet, il semble que c'est le besoin de bouc émissaire qui se reproduit de génération en génération, comme une solution invisible au défi de se positionner dans sa construction identitaire par rapport à l'autre, utilisant des jugements de valeur superficiels (nous sommes supérieurs ou inférieurs à l'autre, en fait, à différents autres).

Derrière des contenus identitaires différents, il s'agit sans doute de la même question du rapport de pouvoir et d'auto-identification de la majorité ancienne par rapport à la plus récente forme d'identité minoritaire. Cette construction identitaire majoritaire doit se positionner face au miroir que représente cette nouvelle minorité d'un point de vue identitaire. Pour ce qui est du Québec, les rapports sont plus complexes, comme dans un quadrilatère invisible qui relie quatre solitudes: les autochtones, les francophones, les anglophones et les différents groupes d'« immigrants», quelle que soit leur diversité. Et pour 
ceux qui ne s'y retrouvent pas parmi les plus récents immigrés, ils doivent s'ajuster dans une certaine mesure et se «fondre» à un des trois autres groupes, le plus souvent par l'entremise de la langue parlée à la maison après une ou deux générations d'intégration ou d'acculturation ou d'assimilation et de mixité ethnique, religieuse, raciale, linguistique, idéologique, etc., selon l'histoire familiale de chacun.

Ces processus dans le rapport à l'autre ne sont bien sûr pas seulement présents chez le groupe identitaire majoritaire, ils existent également à l'intérieur de chaque groupe minoritaire. Dans leur cas aussi, le « "moi" et "nous" » du « je » sujet et objet, agent actif et passif à la fois, se forge au [106] contact des multiples « je » et « nous » rencontrés au fil des jours et des années, en personne et par voix et images interposées, vues, entendues ou lues.

Est-ce que la commission Bouchard-Taylor n'est pas devenue une sorte d'espace public pour l'expression de ces tendances diverses au sein de la société québécoise en pleine transformation, donnant un exemple de démocratie ouverte? Les tensions qui en sont ressorties ont permis aux commissaires de proposer aux politiciens de nouvelles pistes pour favoriser le vivre ensemble au Québec. Mais leurs recommandations seront-elles bien accueillies? S'il semble que plusieurs resteront lettres mortes, quelles seront alors les réactions du public? C'est un défi que d'essayer de mesurer ces réactions pour tous les musulmans du Québec, et pour le plus grand nombre de non-musulmans québécois aussi.

Enfin, une autre piste de réflexion concerne les médias. Plusieurs représentent de nouvelles formes de chaires où les idées de tolérance ou d'intolérance deviennent comme des sermons. Malgré des codes de déontologie très bien définis, la pratique collective de la liberté d'expression et du devoir d'informer le public résulte en un déséquilibre des représentations, effet dont les pratiques individuelles, en elles-mêmes, sont rarement coupables. Ce qui souvent trahit les objectifs louables d'« objectivité », c'est peut-être l'accumulation de représentations «non représentatives» des perceptions majoritaires, malgré la recherche d'un équilibre entre les points de vue. Une simple juxtaposition d'opinions, souvent opposées les unes aux autres, n'y arrive pas. Comment s'assurer, au fil du temps, que ces proportions soient 
bien représentées dans la couverture des événements et des opinions multiples sur tant de sujets variés au sein d'une société en pleine croissance et transformation? Le défi est grand et ne peut être résolu qu'à plusieurs.

\section{Hypothèse de recherche}

\section{Retour à la table des matières}

L'hypothèse de recherche proposée ici est donc la suivante: que le processus d'intégration des musulmans au Québec suit un parcours semblable, en gros, à celui d'autres communautés principalement immigrantes. Dans cette optique, les réactions aux musulmans soulevées dans le contexte de la commission Bouchard-Taylor démontrent la reproduction sociale de discours d'exclusion qui sont présents au sein d'une partie de la société québécoise majoritairement canadiennefrançaise depuis plusieurs générations. La question demeure de savoir jusqu'à quel point le traitement de l'autre se fait par répétition du passé, seule l'identité de l'autre changeant avec le temps. Dans quelle mesure ce qui se passe aujourd'hui par rapport aux musulmans ressemble ou non à ce que d'autres groupes identitaires ont vécu au cours de leur processus d'immigration et d'intégration au Québec? Et que dire des rapports aux autochtones, tant passés que présents? Une analyse approfondie de la commission Bouchard-Taylor pourra répondre à ces questions et évaluer l'hypothèse de [107] recherche. Ce sera aussi le seul moyen d'arriver à une opinion plus équilibrée des perceptions générales de l'impact de cette commission sur les relations entre Québécois d'origine canadienne-française et ceux d'origine ou d'identité musulmane. Une telle recherche contribuera ainsi à éclairer le débat théorique sur la place de la religion dans l'espace public laíque. Pour l'instant, deux scénarios sont envisageables quant à l'impact de la commission Bouchard-Taylor et de ses recommandations générales sur les relations entre «Québécois » et «musulmans » au Québec: celui qui améliore la compréhension mutuelle et celui qui détériore les relations, en permettant aux perceptions négatives d'être propagées médiatiquement beaucoup plus que les perceptions positives. Dans les 
deux cas, son importance dépendra de la façon dont cette commission aura canalisé une crise identitaire de part et d'autre.

Le cas du Québec et de la commission Bouchard-Taylor soulève des questions importantes quant au processus de démocratisation et d'intégration qui se vivent dans toutes les sociétés plurielles. Est-ce que ce genre d'exercice de démocratie publique aide ou non, et dans quelle mesure, les processus d'intégration sociale, surtout par rapport aux identités religieuses dans une société dite laïque? Il faudra analyser les réponses à une telle question pour arriver à savoir si ce genre de commission peut être perçu comme un outil de construction de la paix, dans la mesure où elle aura pu servir de soupape ou d'exutoire pour un public souvent assoiffé d'être vu, d'être entendu, dans une société surmédiatisée. Des recherches plus approfondies permettront de discerner si la commission Bouchard-Taylor a été un mécanisme de prévention de conflits sociaux à grande échelle ou, au contraire, une forme d'opportunité pour la consolidation de débats identitaires où la forte présence de discours d'inclusion fut trop souvent masquée par les opinions et sentiments d'exclusion. Attendons donc que la poussière retombe pour mieux déterminer si la tendance sociale ira vers l'intégration ou vers la ghettoïsation et l'exclusion.

Entre-temps, il est possible de conclure que la commission Bouchard-Taylor fut à la fois un récepteur et un catalyseur des perceptions, surtout en ce qui concerne les relations entre musulmans et nonmusulmans. En étudiant cette commission en profondeur, il sera possible de mettre à l'épreuve l'idée que des déséquilibres dans la gestion des groupes identitaires à l'intérieur de sociétés plurielles peuvent être enracinés dans des strates de privilèges et d'exclusions sociales beaucoup plus anciennes que prévues. Il pourrait s'agir de reproduction d'incohérences et d'un retour d'un besoin de trouver un bouc émissaire, aujourd'hui les musulmans, qui exacerbent en fait les tensions sociales, minant ainsi les croyances, les intensions et les efforts de bonne volonté et de valeurs de justice sociale au sein même de l'idéal qu'une majorité de la population veut véhiculer dans sa propre société. C'est davantage dans cette direction que nos efforts collectifs de mesurer les vrais changements face à l'altérité doivent se concentrer, afin 
qu'un jour une société réellement pluraliste puisse voir le jour, au Québec comme ailleurs.

[139]

\section{Résumé}

Patrice Brodeur

La commission Bouchard-Taylor et la perception des rapports entre «Québécois » et « musulmans » au Québec

\section{Retour à la table des matières}

Cet article aborde des questions théoriques préalables aux recherches sur les perceptions des rapports entre «Québécois» et «musulmans » au Québec à la lumière des événements reliés à la commission Bouchard-Taylor en 2007-2008. Il présente d'abord une balise théorique fondée sur une approche des identités pluriscopes, puis il propose des remarques contextuelles quant à la nature des liens entre laïcité et religion au Québec qui ont influé sur ces perceptions. Pour les travaux ultérieurs, il suggère une hypothèse de travail selon laquelle la diversité de la perception des musulmans au Québec par la majorité non musulmane reproduit un rapport ancien à l'altérité chez les Québécois de souche canadienne-française catholique qui va de la tolérance et de l'ouverture à l'intolérance et à l'exclusion. Ce qu'il reste à déterminer est l'importance relative de ces attitudes les unes par rapport aux autres.

mots clés : identité, Bouchard-Taylor, exclusion, islam, Québec. 


\section{Abstract}

Patrice Brodeur

La commission Bouchard-Taylor et la perception des rapports entre «Québécois » et « musulmans » au Québec

\section{Retour à la table des matières}

This paper deals with theoretical questions for future research on the perceptions of the relations between "Quebecers" and "Muslims" in Quebec, in light of the events surrounding the Bouchard-Taylor Commission. It first presents theoretical guidelines based on the approach of pluriscope identities. This is followed by contextual remarks about the nature of the links between secularism and religion in Quebec, which have influenced such perceptions. A working hypothesis is formulated: the diverse perceptions of Muslims by the non-Muslim French-Canadian majority in Quebec are the results of a reproduction of a pattern of relationships to otherness, which includes a broad range of attitudes, from tolerance and openness to intolerance and exclusion. What remains to be established is the relative importance of these discourses with respect to each other.

keywords : Identity, Bouchard-Taylor, exclusion, Islam, Quebec 


\section{Resumen}

Patrice Brodeur

La commission Bouchard-Taylor et la perception des rapports entre «Québécois » et « musulmans » au Québec

\section{Retour à la table des matières}

Este artículo aborda las preguntas teóricas para la reflexión y las búsquedas futuras sobre las percepciones de las relaciones entre quebequeses y musulmanes en Quebec a la vista de los acontecimientos relacionados con la Comisión Bouchard- Taylor en 2007-2008. El texto presenta primeramente una etiqueta teórica basada en el enfoque de identidades a múltiples perspectivas. Siguen varias observaciones contextuales en cuanto a la naturaleza de los vínculos entre laicidad y religión en Quebec, lazos que han influido estas percepciones. Varias preguntas de reflexión y de búsqueda han sido destacadas para un futuro análisis de esta comisión, asi como una hipótesis de trabajo, es decir : la diversidad de las percepciones por la mayoria no musulmana de los musulmanes en Quebec, son el fruto de una reproducción de la relación entre la alteridad y los Quebequeses de raiz canadiensefrancofona, que incluyen y cubren un abanico de discursos desde la tolerancia hasta los de intolerencia y de exclusion. Lo que queda por determinar es la importancia relativa de estos múltiples discursos, los unos con respecto los otros.

palabras clave : identidad, Bouchard-Taylor, exlusion, islam, Quebec

Fin du texte 